\title{
Emplazamiento, implementación, pruebas de funcionamiento y propuestas de mejora de los sistemas de bombeo mediante rueda hidráulica y ariete multipulsor para el abastecimiento de agua para irrigación en la localidad del campus Juan Lunardi - Yumacay - Paute - Azuay
}

\author{
Nelson Jara Cobos*, Edgar Campoverde Ochoa**, Damián Pizarro Jara** \\ email: njara@ups.edu.ec
}

\section{Resumen}

Para la implementación de los prototipos de sistemas de bombeo se requirió del diseño y la construcción de un pozo desarenador y de un sistema de tuberías, tanto para la toma como la descarga de los dos sistemas de bombeo.

Posteriormente a la implementación se procedió con las pruebas de campo, teniendo como referencia los resultados óptimos y recomendados por los constructores, los mismos que fueron producto de las pruebas de laboratorio.

En cuanto al ariete hidráulico se realizaron ocho pruebas, las mismas que comprobaron que mientras más altura de bombeo exista el caudal y el rendimiento van decreciendo, hasta que finalmente el ariete se detiene, teniendo como límites de funcionamiento una altura máxima de bombeo de 70 metros con un caudal de $0,2 \mathrm{l} / \mathrm{min}$ y un caudal máximo de 4 1/min a una altura de 42 metros.

En cuanto a la rueda hidráulica se realizó dos pruebas generales en diferentes direcciones de chorro, a $45^{\circ}$ y chorro recto, presentando una mayor eficiencia la cuando se utiliza a $45^{\circ}$, con el cual se obtuvo $34 \mathrm{rpm}$ en la rueda y un caudal bombeado de 4,2 1/ min a $\mathbf{1 2 0}$ metros de altura, mientras que el chorro recto permitió girar a la rueda a $66 \mathrm{rpm}$ dándonos un caudal de $8 \mathrm{l} / \mathrm{min}$ a 120 metros de desnivel.

\begin{abstract}
For the implementation of the prototype pumping systems are required for the design and construction of a sludge trap and a system of pipes, both for taking and discharge of the two pumping systems.
\end{abstract}

Following the implementation proceeded with the field tests, taking as reference the optimal and recommended by the manufacturers, they were the result of laboratory tests.

As for the bydraulic ram eight tests were conducted, they found that the more you pump up the volume and there are decreasing performance until finally the ram stops, with the operating limits of a maximum pumping height of 70 meters with a flow rate of $0.2 \mathrm{l} /$ min and a maximum flow of $4 \mathrm{l} / \mathrm{min}$ at a beight of 42 meters.

As for the waterwheel was made two general tests in different directions, jet, jet at $45^{\circ}$ and straight, with a bigher efficiency when using the jet to $45^{\circ}$, which was $34 \mathrm{rpm}$ at the wheel and pumped flow rate of $4.2 \mathrm{l} / \mathrm{min}$ to 120 meters, while the straight stream allowed to turn the wheel at $66 \mathrm{rpm}$ giving a flow rate of $8 \mathrm{l} / \mathrm{min}$ to 120 meters of altitude.

Keywords: Pumping, multipulsor ram, water wheel, sand trap

Palabras clave: Sistemas de bombeo, ariete multipulsor, rueda hidráulica, desarenador.

\footnotetext{
* Ingeniero Mecánico, Director de la Carrera de Ingeniería Mecánica - UPS - sede Cuenca, estudiante de Maestría en automatización y control industrial UPS.

*** Estudiantes de la Carrera de Ingeniería Mecánica - UPS - sede Cuenca. 


\section{Introducción}

Dos proyectos realizados en la Universidad Politécnica Salesiana, sede Cuenca en el área de las energías alternativas, se centran básicamente en dar una solución al problema de dotación de agua para riego agrícola a bajo costo [1], mediante la explotación de energías limpias, en nuestro caso la energía que se aprovecha es la hidráulica. Los mencionados proyectos se estudiaron, diseñaron y construyeron hace aproximadamente dos años.

- Diseño de un prototipo de bomba de ariete hidráulico multipulsor de abastecimiento de agua para irrigación [3].

- Diseño y Construcción de una rueda hidráulica para el abastecimiento de agua para riego a una altura de 120 metros [10].

\subsection{Sistema de bombeo por bomba de ariete hidráulico multipulsor}

El ariete hidráulico consiste en una máquina que aprovecha únicamente la energía de un pequeño salto de agua para elevar parte de su caudal a una altura superior; en este proceso, el sistema pierde agua para compensar la altura que eleva [7].

Funcionamiento. El agua se acelera a lo largo del tubo de alimentación hasta alcanzar una velocidad suficiente como para que se cierre la válvula de impulsión, entonces se crea una fuerte presión, al detenerse el agua bruscamente. Este golpe de presión abre la válvula de retención y hace pasar un pequeño chorro de agua a la cámara de aire, hasta que se equilibran las presiones [3]. En ese momento la gravedad abre la válvula de impulsión y se cierra la de retención, repitiéndose de nuevo el ciclo. El agua, a cada golpe de aire, hace fluir con continuidad por la manguera de elevación. El ritmo de golpes por segundo suele ser de uno o dos.

\subsection{Sistema de bombeo por rueda hidráulica}

La rueda hidráulica es un dispositivo que capta energía de una corriente de agua y la transforman en trabajo mecánico.

Una rueda hidráulica está constituida por paletas (álabes), colocada con su eje horizontal [10]. El caudal de agua conducido por una tubería ejerce una fuerza cuando se impacta con cada paleta, produciendo la rotación de la rueda y un torque debido a la fuerza; a medida que las rpm se incrementan también el torque crece debido a la inercia de la masa; este torque total es aprovechado para mover sistemas mecánicos de bombeo como un pistón, que servirá plenamente para bombear agua [12].

\section{Procedimientos y resultados}

\subsection{Diseño del parque hidráulico}

\subsubsection{Localización y análisis del lugar en donde se realizará la implementación de los equipos}

Las características que posee la localidad de Yumacay - Paute, ubicada en la provincia del Azuay, junto al campus Juan Lunardi de la UPS; sobre todo, por contar con una fuente de agua constante en el año, una laguna artificial de $40000 \mathrm{~m} 3$ y poseer pendientes favorables para el funcionamiento de los equipos, fue determinante para su emplazamiento [10], como se puede observar en la figura 1 . 


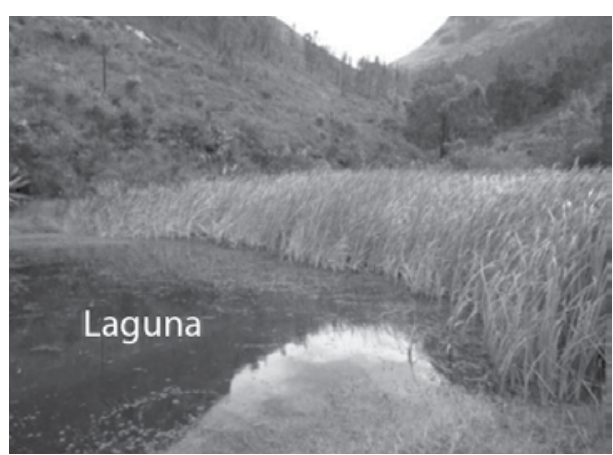

Fig. 1. Fuente de alimentación

El sitio en mención cumple con lo establecido a continuación:

- Espacio suficiente para la construcción de las obras civiles requeridas.

- Existe un desnivel de terreno necesario para cumplir los requerimientos de los equipos.

- Se puede conducir fácilmente el agua utilizada por los equipos a su cauce natural.

La zona utilizada para el montaje de los equipos de acuerdo a las características establecidas se encuentra a 30 metros hacia abajo de la laguna artificial, se puede observar en la figura 2.

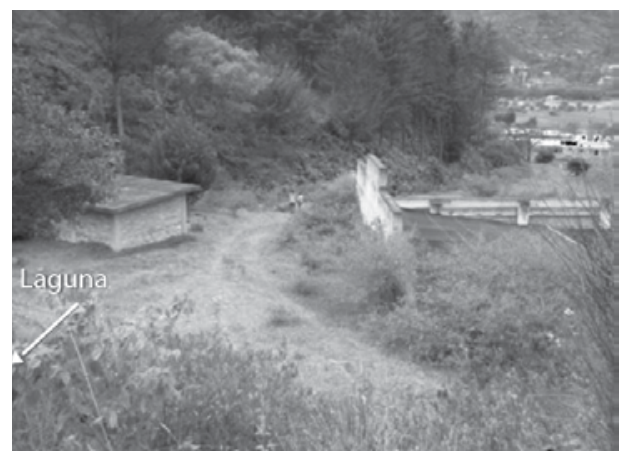

Fig. 2. Lugar para la implementación.
En las figuras 3 y 4 se muestra un bosquejo en donde se indica la posible posición de cada una de las diferentes fases del proyecto a ser construidos.

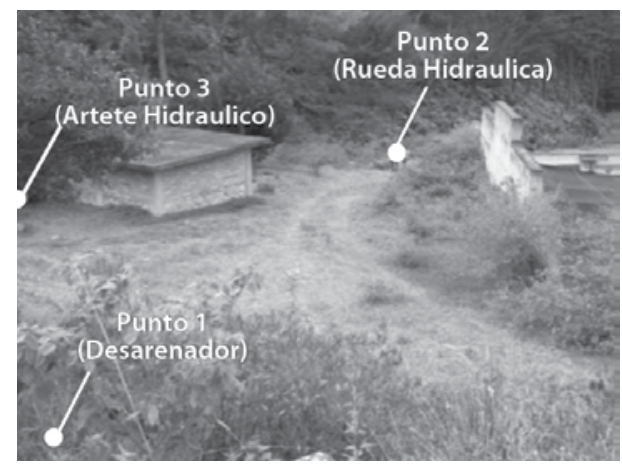

Fig. 3. Posible ubicación del proyecto planteado

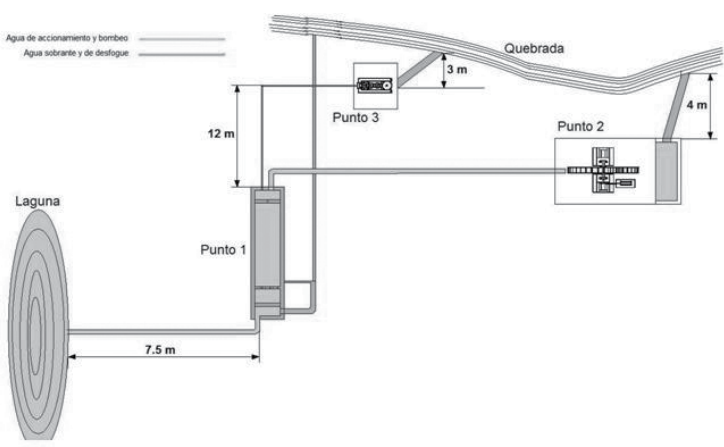

Fig. 4. Distribución general del parque hidráulico

\subsubsection{Diseño del desarenador}

Generalmente, la captación de una fuente superficial permite el paso de material de cierto tamaño, sobre todo en época lluviosa, lo cual arruinaría el sistema o provocaría desajustes por obstrucción en muy corto tiempo, es por eso que se requiere construir un desarenador [6].

En nuestro caso, el material a sedimentar es una arena fina cuyo diámetro la hemos tomado 
como $0,01 \mathrm{~cm}$, esto debido a que el agua de alimentación es obtenida de una laguna y por ende esta tiene la función de un gigantesco desarenador «natural».

Por el material a sedimentarse y el caudal que circulará, se seleccionó un desarenador de tipo convencional como se muestra en la figura 5.

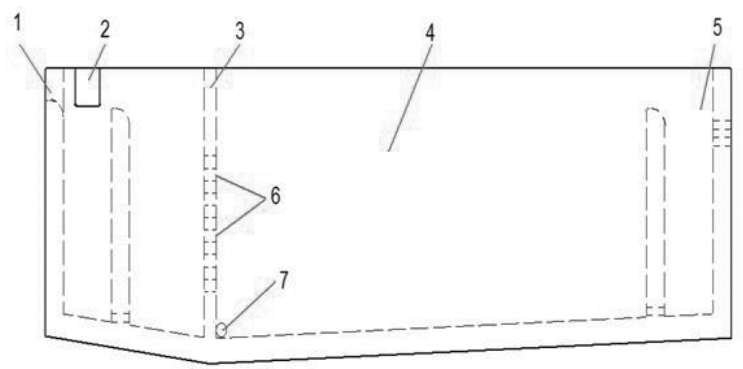

Fig. 5. Desarenador convencional, partes principales

1. Zona de entrada

2. Vertedero de alivio

3. Placa difusora

4. Zona de sedimentación

5. Zona de salida

6. Agujeros

7. Evacuación de lodo

\subsubsection{Diseño de la distribución de agua (red de tuberías) [2]}

Para iniciar con el diseño de la distribución de tuberías, es necesario analizar la naturaleza del sitio en donde se realizará la instalación, ya que existen varios desniveles en el terreno y eso implica la utilización de varios accesorios en la red de tuberías, los mismos que causan el cambio de dirección del flujo ocasionando pérdidas en la presión [11].

En las figuras 6 y 7 se muestran la forma y las dimensiones finales con las cuales se instalarán la toma de carga de cada uno de los sistemas de bombeo.

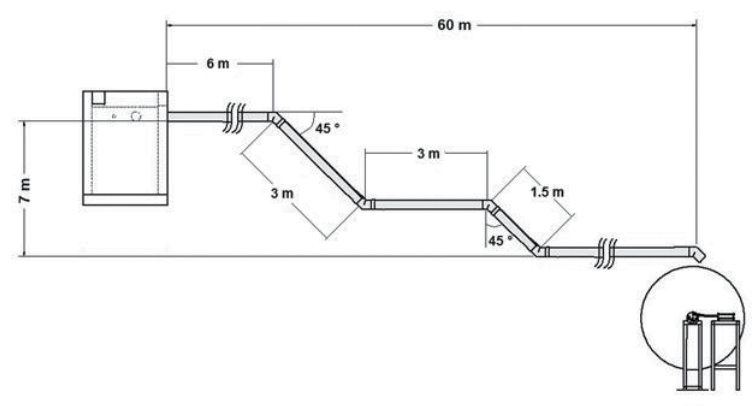

Fig. 6. Longitud de tubería y desnivel de las instalaciones de la rueda hidráulica

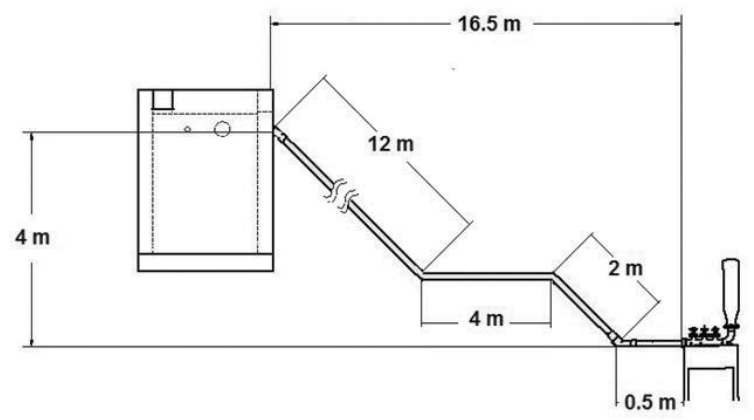

Fig. 7. Longitud de tubería y desnivel de las instalaciones del ariete

\subsubsection{Implementación del parque hidráulico}

La implementación del parque hidráulico nos permitirá comprobar el funcionamiento y los rendimientos reales de los prototipos estudiados, para lo cual en primera instancia se construyó toda la obra civil que fue diseñada (figura 8).

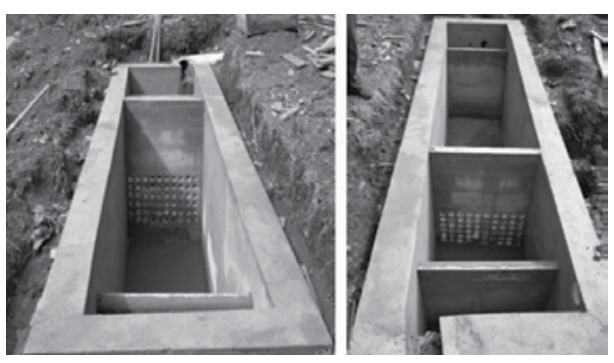

Fig. 8. Desarenador 
El montaje de la rueda hidráulica y el ariete multipulsor se pueden observar en las figuras 9 y 10.

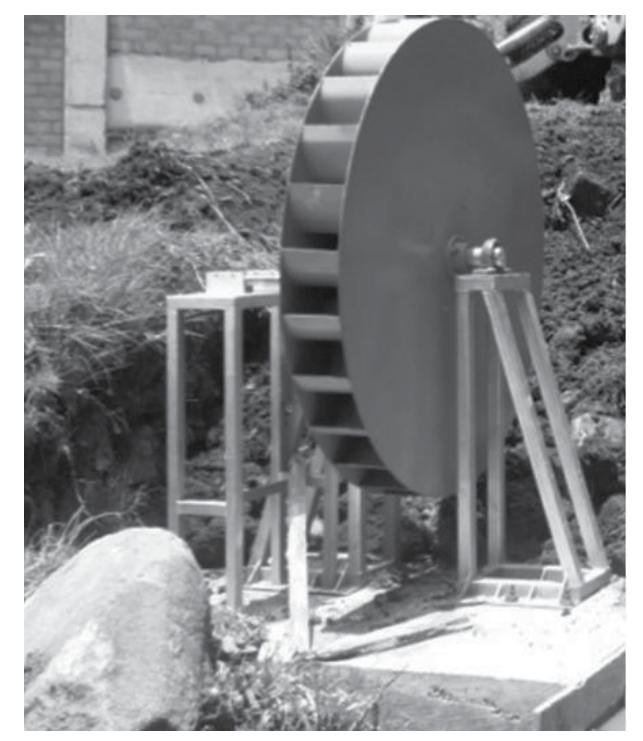

Fig. 9. Rueda hidráulica

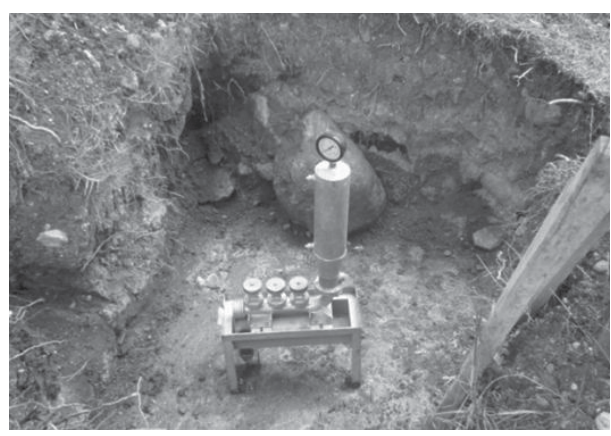

Fig. 10 Ariete hidráulico

Para controlar el paso del flujo desde la toma de agua en la laguna hasta el desarenador, se requiere de una compuerta deslizante (figura 11).

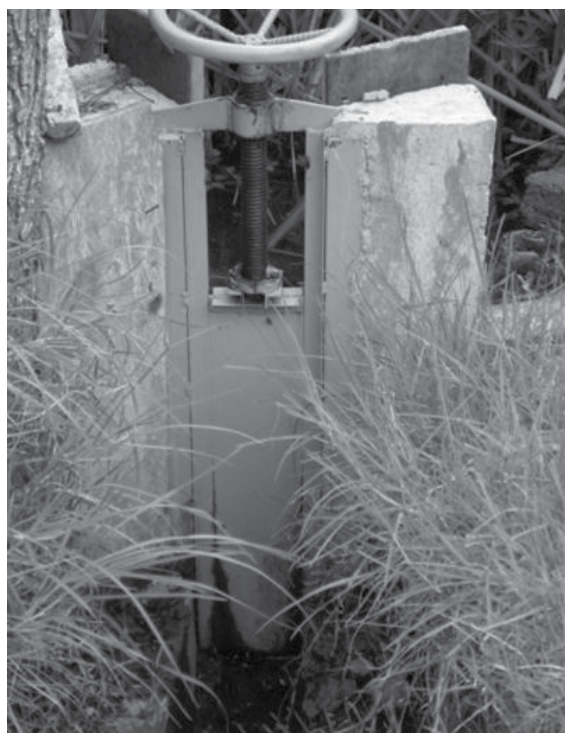

Fig. 11. Compuerta deslizante

\subsection{Pruebas de funcionamiento}

Se procedió a realizar las respectivas pruebas para el funcionamiento de los sistemas in situ y su correspondiente comparación de los resultados, con los obtenidos en las pruebas de laboratorio que se realizaron en los proyectos iniciales [3][10].

\subsubsection{Prueba de funcionamiento del sistema de bombeo por ariete hidráulico}

\section{Pruebas de operación}

A continuación en la tabla 1 se recopiló todos los resultados de las pruebas tanto de laboratorio como las de campo, para luego hacer una comparación de las mismas. 
Tabla 1. Resultados de las pruebas tanto de laboratorio como las de campo

\begin{tabular}{|c|c|c|c|c|c|c|c|}
\hline \multicolumn{8}{|c|}{ RESULTADO DE LAS PRUEBAS } \\
\hline \multicolumn{4}{|c|}{ CAMPO } & \multicolumn{4}{|c|}{ LABORATORIO } \\
\hline $\begin{array}{c}\text { \# de } \\
\text { prueba }\end{array}$ & $\begin{array}{c}\text { Altura } \\
\text { de } \\
\text { bombeo } \\
\text { (m) }\end{array}$ & $\begin{array}{c}\text { Caudal } \\
\text { de } \\
\text { entrega } \\
\text { (1/dia) }\end{array}$ & $\begin{array}{c}\text { Rendimiento } \\
(\%)\end{array}$ & $\begin{array}{c}\text { \# de } \\
\text { prueba }\end{array}$ & $\begin{array}{c}\text { Altura } \\
\text { de } \\
\text { bombeo } \\
\text { (m) }\end{array}$ & $\begin{array}{c}\text { Caudal } \\
\text { de } \\
\text { entrega } \\
\text { (V/dia) }\end{array}$ & $\begin{array}{c}\text { Rendimiento } \\
(\%)\end{array}$ \\
\hline 1 & 40 & 1440 & 3.05 & 19 & 40 & 264 & $0,746 \%$ \\
\hline 2 & 50 & 720 & 1.907 & 20 & 50 & 228 & $0,806 \%$ \\
\hline 3 & 55 & 360 & 1.049 & 21 & 55 & 302 & $1,174 \%$ \\
\hline 4 & 85 & 0 & 0 & 22 & 85 & 140 & $0,841 \%$ \\
\hline 5 & 90 & 0 & 0 & 23 & 90 & 95 & $0,604 \%$ \\
\hline 6 & 0 & 0 & 0 & 24 & 0 & 0 & $0,000 \%$ \\
\hline
\end{tabular}

Como se puede observar en los tres primeros resultados de las pruebas de campo existe una mayor cantidad tanto en caudal de entrega como en rendimiento con respecto a las pruebas de laboratorio [3].

Una de las razones del porqué hay una diferencia entre los resultados es debido a que en el sitio se instaló con un desnivel de 4 metros mientras que las pruebas se realizaron a 3 metros de desnivel.

Lo contrario sucede en las siguientes tres pruebas, ya que en estos casos no se logró obtener caudal de entrega en ninguna, este problema podría estar basado en las pérdidas existentes en el conducto de entrega, ya que la distancia existente entre el punto de descarga y el ariete es de 181,2 metros, a estos tenemos que añadir los 10 metros que se implementó en el primer tramo para lograr llegar al primer punto de descarga, además de las uniones que existen entre tramo y tramo, entonces con esto tenemos una distancia de 191,2 metros de manguera utilizada, como en los resultados de laboratorio se puede observar el caudal de entrega es demasiado bajo $(0,098 \mathrm{l} / \mathrm{min})$ para circular por esta longitud de manguera y además venciendo un desnivel de 85 y 90 metros.

En las pruebas de campo, además, se logró verificar que la presión de bombeo llegaba a un valor máximo de 7 Bar, entonces las pruebas de laboratorio se realizaron basándose en esta presión, por lo que según esto, la altura máxima de bombeo seria 70 metros; por esta razón se realizaron dos pruebas más, la una para encontrar el punto en donde se tendría el caudal de entrega máximo y la otra para encontrar el punto en donde se obtendría la altura máxima, teniendo en cuenta los límites antes estudiados que van desde los 40 a los 90 metros de desnivel, los pesos de 500 a $1500 \mathrm{~g}$ y las carreras de 3 a $9 \mathrm{~mm}$.

\section{Localización del caudal máximo de entrega}

Para la localización de este punto se establecieron distintos valores de carrera y peso del ariete, teniendo como base los $3 \mathrm{~mm}, 500 \mathrm{~g}$ y 
40 metros de desnivel, con esto se procedió a incrementar la carrera y el peso en cada válvula e ir subiendo el punto de descarga, realizando las respectivas pruebas del caudal de entrega.

Se logró, finalmente, establecer el caudal máximo que puede entregar el ariete hidráulico, las características de regulación en el prototipo y los resultados de esta prueba se muestran a continuación en la tabla 2 .

Tabla 2. Resultados caudal máximo

\begin{tabular}{|c|c|c|c|c|c|c|c|c|}
\hline \multicolumn{9}{|c|}{ PRUEBA DE CAMPO (Caudal máximo) } \\
\hline $\begin{array}{l}\text { \#Valvulas } \\
\text { activas }\end{array}$ & "Válvula & $\begin{array}{c}\text { Carrera } \\
(\mathrm{mm})\end{array}$ & $\begin{array}{c}\text { Peso } \\
\text { (gx) }\end{array}$ & $\begin{array}{l}\text { Altura } \\
\text { bombeo } \\
\text { (m) }\end{array}$ & $\begin{array}{l}\text { Caudal } \\
\text { Entrega } \\
\text { (timinin) }\end{array}$ & $\begin{array}{c}\text { Caudal } \\
\text { Entrega } \\
\text { (tt/da) }\end{array}$ & No Golpes & $\begin{array}{l}\text { Presión } \\
(\mathrm{mH} 2 \mathrm{O})\end{array}$ \\
\hline \multirow{3}{*}{3} & 1 & 7 & 1500 & \multirow{3}{*}{42} & \multirow{3}{*}{4} & \multirow{3}{*}{5760} & \multirow{3}{*}{40} & \multirow{3}{*}{50.99} \\
\hline & 2 & 5 & 1500 & & & & & \\
\hline & 3 & 3 & 1500 & & & & & \\
\hline
\end{tabular}

Como se puede observar los resultados obtenidos se lograron gracias a las diferencias entre las carreras en cada válvula y el peso constante de $1500 \mathrm{~g}$, con esto tenemos un caudal impresionantemente grande con respecto a las pruebas anteriores, en cuanto a su eficiencia se logró obtener de igual manera un valor alto de $12,82 \%$.

\section{Altura máxima de entrega}

Se logró situar el punto máximo de altura de bombeo, el mismo que se encuentra ubicado en las coordenadas escritas en la tabla 3.

Tabla 3. Coordenadas del punto de caudal máximo

\begin{tabular}{|l|l|l|}
\hline Variante & Coordenadas & Orientación \\
\hline Longitud & $78,755894^{\circ}$ & Oeste \\
\hline Latitud & 2,772035 & Sur \\
\hline Altitud & $2301,1 \mathrm{msnm}$ & \\
\hline
\end{tabular}

\subsubsection{Pruebas de funcionamiento del sistema de bombeo por rueda hidráulica}

Estas pruebas de basaron en los resultados de los diferentes modelos estudiados en un banco de pruebas construido específicamente para este proyecto (figura 12), de acuerdo a lo establecido, el prototipo es factible para bombear agua a $120 \mathrm{~m}$ de altura con un caudal de 0,17 l/s [10].

BANCO DE PRUEBAS

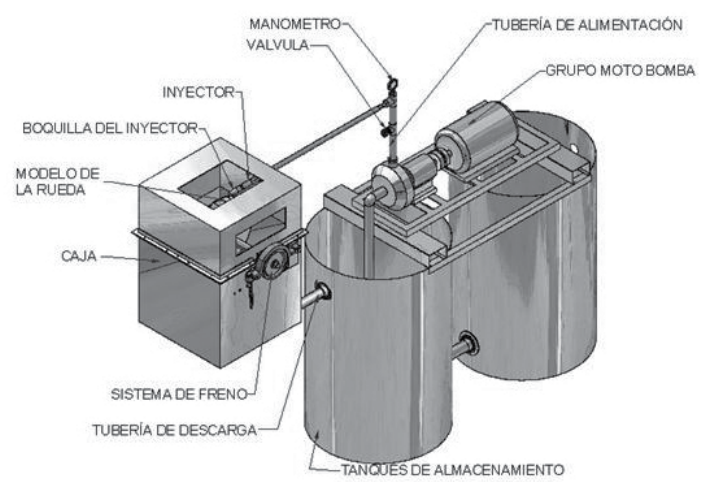

Se realizó la prueba con las condiciones planteadas en los resultados de los estudios en laboratorio, es decir intentaremos bombear agua a 120 metros de altura con un caudal 0,17 1/s y dependiendo de los resultados de la prueba se variará la altura para comprobar su capacidad máxima.

\section{Prueba de campo}

Una vez ubicada la dirección del chorro de agua de una forma directa hacia los álabes de la rueda con la finalidad de incrementar el número de revoluciones de la rueda y por ende su fuerza centrífuga, se logró obtener un mayor caudal de entrega (figuras 13 y 14). 


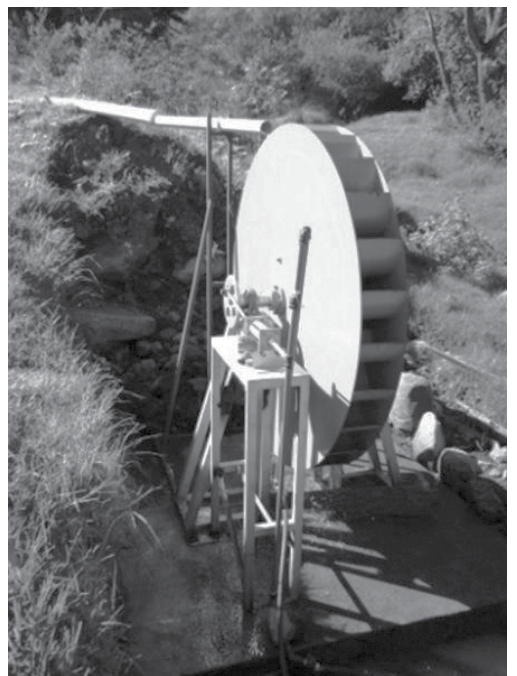

Fig. 13. Ubicación del chorro de carga

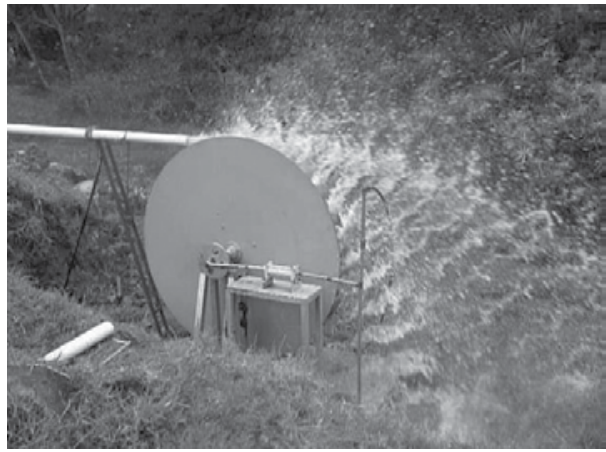

Fig. 14. Prueba de campo

Los resultados de las pruebas se describen en la tabla 4.

\begin{tabular}{|c|c|c|c|c||}
\hline \multicolumn{5}{|c||}{ PRUEBA DE CAMPO \#2 } \\
\hline \# Prueba & $\begin{array}{c}\text { Diámetro del } \\
\text { chorro }(\mathrm{mm})\end{array}$ & $\begin{array}{c}\text { Altura bombeo } \\
(\mathrm{m})\end{array}$ & $\begin{array}{c}\text { Caudal Entrega } \\
\text { (lt/min) }\end{array}$ & $\begin{array}{c}\text { Revoluciones de } \\
\text { la rueda }\end{array}$ \\
\hline 1 & 70 & 100 & 3 & 66 \\
\hline
\end{tabular}

Tabla 4. Resultados de la prueba de campo (rueda hidráulica a 100 metros, chorro directo) 2.2 Propuestas de mejoras para los sistemas de bombeo

Las siguientes consideraciones tienen por objetivo eliminar algunos inconvenientes que se presentaron al momento del funcionamiento de cada uno de los sistemas de bombeo para su posterior implementación y elevación de mayor cantidad de agua posible.

\subsubsection{Propuesta de mejora para sistema de bombeo por ariete hidráulico}

1. Acople. La propuesta sería remplazar este acople por una brida, que resulta mucho más eficiente, su posible implementación se puede apreciar en la figura 15.

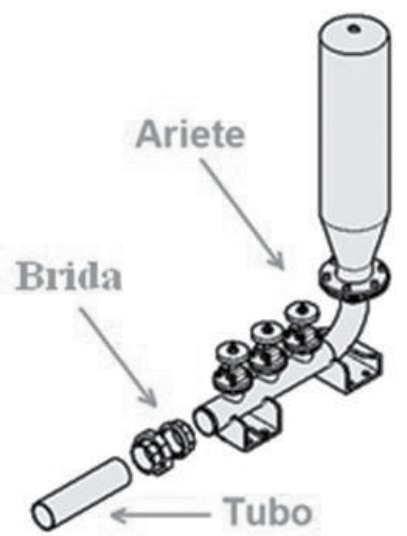

Fig. 15. Montaje de la mejora (brida)

2. Válvulas. Para mejorar el desempeño mecánico del ariete se pretende diseñar un elemento que proporcione la fuerza necesaria y mantenga el golpe de ariete en la bomba, una de las partes del ariete es el soporte de la válvula de impulsión (figura 16).

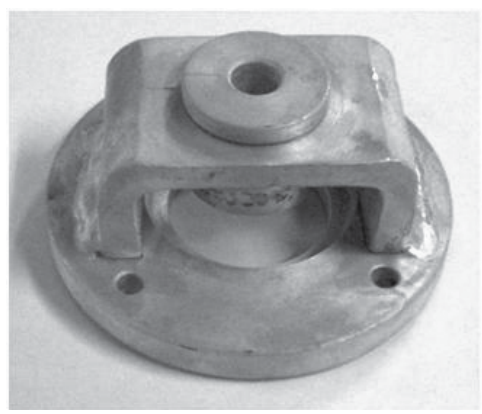

Fig. 16. Soporte válvula de impulsión 
Dado que este mecanismo presenta la mayor cantidad de inconvenientes, se lo puede reemplazar por uno de resortes, los mismos que otorgan la fuerza de impulso para que la válvula permanezca abierta cuando el golpe de ariete no influya y de igual forma cuando la presión del agua esté al máximo lo comprima (figura 17).

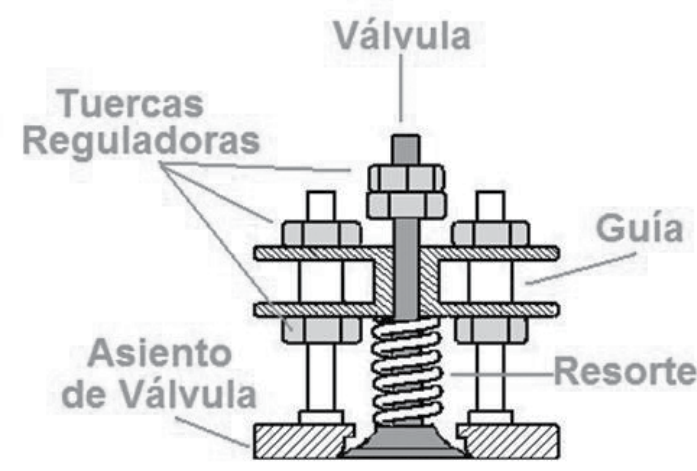

Fig. 17. Sistema propuesto para mejorar el funcionamiento en las válvulas

$\mathrm{Al}$ igual que en el sistema implementado por pesas (figura 16), este también presenta dos posiciones de las válvulas (figura 18).
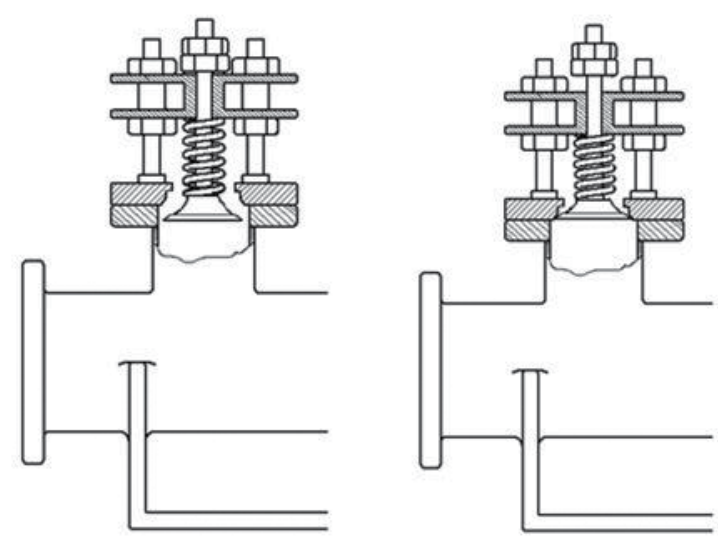

Fig. 18. Funcionamiento del sistema de resortes
3. Válvula check en la cámara de aire. La solución a este inconveniente sería el reemplazo de la unión roscada por una tercera brida, la misma que iría implementada en la mitad, es decir entre el cuerpo del ariete y la cámara de aire, como se muestra en la figura 19.

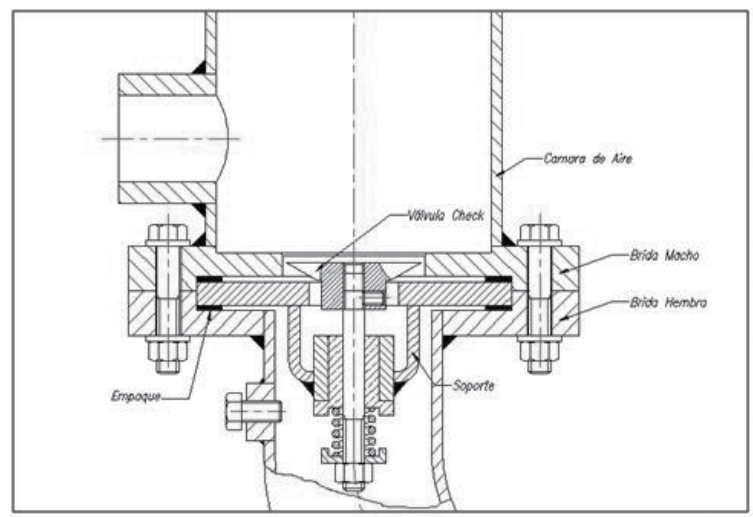

Fig. 19. Propuesta de mejora de la conexión de la válvula check.

4. Cámara de aire. En la parte superior del interior de la cámara se encuentra aire en condiciones estáticas. Si la cámara de aire se llena completamente de agua, el ariete puede detenerse o llegar a romperse, por lo que la cámara de aire debe tener un tamaño adecuado, además para mejor funcionalidad debe tener un tapón de drenaje en su parte inferior y un respiradero ubicado antes de la válvula check para recargar el aire que se pierde al bombear el agua, y permita regular la entrada de aire.

Los resultados obtenidos en el cálculo del volumen de la cámara de aire, con el diámetro actual de 0,127 $\mathrm{m}$, se requiere una longitud de $0,73 \mathrm{~m}$; es decir, necesitaríamos incrementar 0,2 $\mathrm{m}$ de longitud a la cámara de aire para alcanzar los $100 \mathrm{~m}$ de altura. 
2.2.4 Propuesta de mejora para sistema de bombeo por rueda hidráulica

1. Mejora del sistema de bombeo. Aunque se obtuvo resultados satisfactorios en las pruebas de campo, pudimos observar que el chorro de descarga es intermitente debido a que posee un solo pistón, para obtener un chorro continuo en la descarga y además mejorar el rendimiento de la rueda es implementar otra bomba de émbolo que funcionen simultáneamente (figura 20).

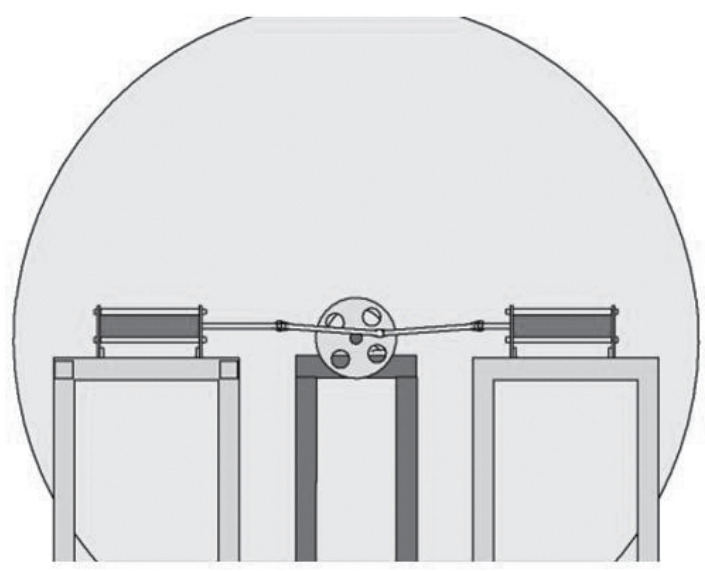

Fig. 20. Rueda hidráulica con dos pistones para bombeo

En cuanto al requerimiento del torque necesario para mover los pistones versus el que se genera en la rueda, se ha verificado que es suficiente, teniendo que lo que se genera supera ampliamente: 5131,686 Nm > 318,6201 Nm.

2. Sistema de succión e impulsión. La succión y la impulsión se dan cuando la rueda gira una revolución, aquí ocurre que las válvulas check se cierran y se abren alternativamente, permitiendo que el agua sea empujada por el pistón hacia la tubería de descarga; esto sucede en cada revolución que da la rueda y por cada pistón. El sistema de dos pistones funciona simultáneamente pero la succión y la impulsión irán alternándose, permitiendo así un flujo constante en la descarga, además de duplicar el caudal de entrega [5], (figura 21).
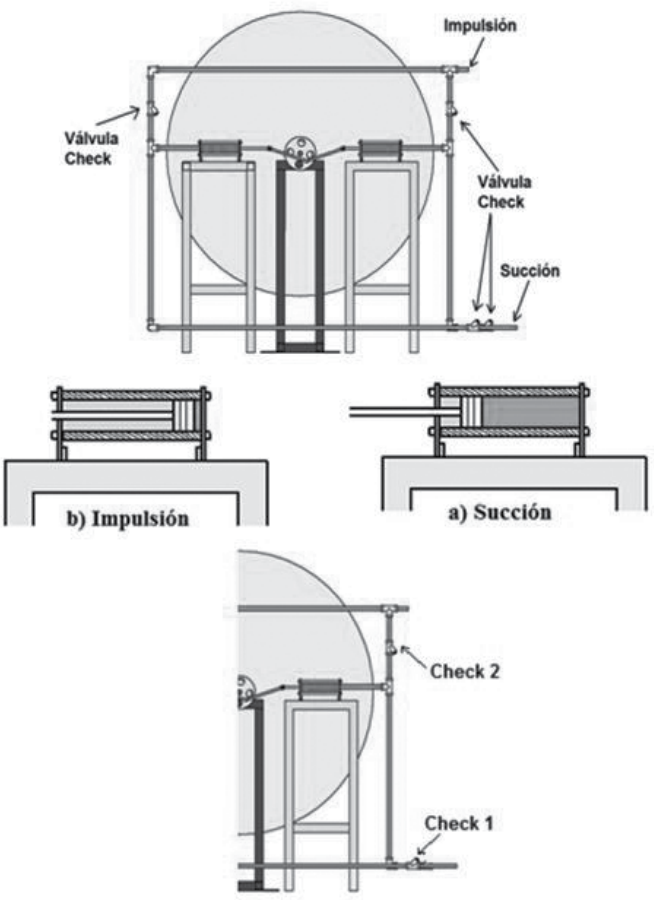

Fig. 21. Sistema de succión e impulsión del agua

\section{Conclusiones}

En la actualidad es menester buscar nuevas formas de energía renovables para evitar continuar contaminando más y más al planeta, este proyecto de implementación es una pequeña contribución para fomentar el desarrollo tecnológico en este campo.

Uno de los principales objetivos de este proyecto fue la implementación de las bombas, específicamente para la irrigación en el sector y poder abastecer de una forma ininterrumpida el líquido vital.

La construcción en un desarenador es indispensable para el funcionamiento de las bombas, ya que sin este dispositivo se reduciría drásticamente la vida útil de los pistones, al lle- 
Debido a la inercia producida por la rueda hidráulica la base del pistón no soportó y cedió, ya que no se encontraba bien realizada la soldadura, por lo que se tuvo que reforzar la estructura.

Durante el funcionamiento de la rueda hidráulica, la bomba de émbolo fue perdiendo presión por el desgaste presente en el cilindro, el mismo que es de PVC, material que no soporta fricción, por lo que fue necesario el rectificado del cilindro para continuar con las pruebas.

Se pudo notar que mientras mayor carrera existía en las válvulas, el golpe es más fuerte y por ende las pesas tienden a deformarse por ser de material maleable (plomo), causando que estas se aflojen y perdiendo así la fuerza del golpe de ariete hasta detenerse.

De las ocho pruebas de campo realizadas en el ariete hidráulico, (prueba 1, 2, 3, 7, 8) se obtuvieron resultados óptimos de funcionamiento tomando en cuenta que en las pruebas 7 y 8 se consiguió el caudal y altura máximo de bombeo, respectivamente; mientras tanto que en las pruebas de campo 4, 5 y 6 no se lograron obtener resultados positivos, esto debido a que la altura de bombeo es demasiadamente grande y no logra vencer la presión que ejerce en los conductos.

En lo que concierne a la rueda hidráulica se realizaron primeramente dos tipos de pruebas referentes a la posición del chorro, la primera a $45^{\circ}$ obteniendo resultados positivos con 34 $\mathrm{rpm}$ de la rueda y un caudal de entrega de 1,8 $1 /$ min a 100 metros de altura, mientras que en la segunda posición mediante el chorro tangente a la rueda, es decir, sin la utilización de ningún accesorio para dirigir el chorro, obteniendo $66 \mathrm{rpm}$ y un caudal de entrega de $3 \mathrm{l} /$ min a 100 metros de altura.
Para el funcionamiento continuo de la bomba de émbolo es preciso realizar el cambio de material en el cilindro, por un material más duro (acero inoxidable) resistente a la fricción y a la corrosión, con esto garantizaríamos el perfecto funcionamiento y alargaríamos la vida útil de la bomba.

El montaje de la tubería de carga en el ariete hidráulico fuera más eficaz si se implementa una brida evitando así inconvenientes en el momento de implementar el ariete con la tubería de carga.

El sistema de resortes propuesto para el funcionamiento de las válvulas evitaría la necesidad de las pesas y sobretodo el desmontaje para poder variar la carrera de las válvulas de impulsión.

En cuanto a la cámara de aire se pudo comprobar que el dimensionamiento depende directamente de la altura y caudal de entrega, pudiendo comprobar que para alcanzar la meta propuesta de bombear agua a los 100 metros de altura con un caudal de $0,2 \mathrm{l} / \mathrm{min}$ es necesario incrementar $20 \mathrm{~cm}$ de longitud en la cámara ya construida. 
Para la mejora de la rueda hidráulica se debe implementar una bomba de embolo adicional, así obtendríamos un incremento en el caudal y en el rendimiento además de un flujo constante de entrega.

\section{Referencias bibliográficas}

[1] Taller de Investigación Alternativa «José Manuel Jiménez».

[2] Arocha. 1978. Abastecimiento de agua, Teoría y diseño.

[3] Bustamante A., Quezada M. E. 2009. Estudio de los parámetros de funcionamiento de la bomba de ariete hidráulico multipulsor AyM 1.0. Universidad Politécnica Salesiana. Facultad de Ingenierías. Carrera de Ingeniería Mecánica. Cuenca.

[4] Cavero, P. (s. f.). En línea: http://www.slideshare.net/rcavero/bomba-de-ariete-pablo-cavero. Obtenido de http://www.slideshare.net/rcavero/bomba-de-ariete-pablo-cavero.

[5] Claudio, M. 2007. Mecánica de fluidos y máquinas hidráulicas. México.

[6] Cuella. Elemento de diseño para acueductos y alcantarillado.
[7] Herrera, L. F. 2005. Tesis, Diseño y construcción de un ariete y de una rueda hidráulica para bombeo de agua en la granja experimental San Marcos del HCPP. Sangolquí.

[8] Espinosa M. J. 2011. Tesis, Planificación y diseño de un sistema hidráulico para el abastecimiento de agua en el barrio San Miguel de Paquiestancia del cantón Cayambe ubicado a $3200 \mathrm{msnm}$. Quito.

[9] Potter, M. C. 2002. Mecánica de fluidos. International Thomson Editores. México.

[10] Saquicela F. A., Velepucha I. R. 2008. Diseño y construcción de una rueda hidráulica para el abastecimiento de agua para riego a una altura de $\mathbf{1 2 0}$ metros. Universidad Politécnica Salesiana. Facultad de Ingenierías. Carrera de Ingeniería Mecánica. Cuenca.

[11] Streeter, V. L. 2005. Mecánica de fluidos. McGraw-Hill.

[12] Vivanco, R. A. 2006. Tesis, Diseño y construcción de un sistema de rueda hidráulica-bomba. Quito. 